\title{
FINITE DIFFERENCE APPROXIMATIONS FOR A CLASS OF NON-LOCAL PARABOLIC EQUATIONS
}

\author{
YANPING LIN, SHUZHAN XU \\ Department of Mathematical Sciences \\ University of Alberta \\ Edmonton, Alberta \\ Canada T6G 2G1 \\ and \\ HONG-MING YIN \\ Department of Mathematics \\ University of Notre Dame \\ Notre Dame, Indiana 46556-0398 \\ (Received October 4, 1994)
}

\begin{abstract}
In this paper we study finite difference procedures for a class of parabolic equations with non-local boundary condition. The semi-implicit and fully implicit backward Euler schemes are studied. It is proved that both schemes preserve the maximum principle and monotonicity of the solution of the original equation, and fully-implicit scheme also possesses strict monotonicity. It is also proved that finite difference solutions approach to zero as $t \rightarrow \infty$ exponentially. The numerical results of some examples are presented, which support our theoretical justifications.
\end{abstract}

KEYWORDS AND PHRASES: Finite difference, non-local, monotonicity, decay, stability, maximum principle.

1991 AMS SUBJECT CLASSIFICATION CODES: 65N30, 45K05, 65M60.

\section{INTRODUCTION.}

In this paper we study finite difference approximations to the solution of the following parabolic equations with non-local boundary condition:

$$
\begin{array}{rlrl}
u_{t}-\Delta u & =0 \text { in } Q_{T}, & \\
u(x, y, 0) & =\phi(x, y), & & (x, y) \in \Omega \\
u(x, y, t) & =\int_{\Omega} K(x, y, \xi, \eta) u(\xi, \eta, t) d \xi d \eta, & & \text { on } \partial \Omega \times[0, T)
\end{array}
$$

where $Q_{T}=\Omega \times(0, T), \quad T>0, \Omega=(0,1) \times(0,1), \phi(x, y) \not \equiv 0$ and $K(x, y, \xi, \eta)$ are known functions. In addition, it is assumed that for some constant $0<\rho<1$ the kernel $K(x, y, \xi, \eta)$ satisfies

$$
\int_{\Omega}|K(x, y, \xi, \eta)| d \xi d \eta \leq \rho<1, \quad \forall(x, y) \in \partial \Omega .
$$


In $[4,5]$ Day considered the one-dimensional problem on $(-L, L), L>0$, with the boundary conditions

$$
u(-L, t)=\int_{-L}^{L} f_{1}(x) u(x, t) d x \quad \text { and } \quad u(L, t)=\int_{-L}^{L} f_{2}(x) u(x, t) d x,
$$

and showed that if

$$
\int_{-L}^{L}\left|f_{1}(x)\right| d x<1 \quad \text { and } \quad \int_{-L}^{L}\left|f_{2}(x)\right| d x<1
$$

then, for the solution $u$,

$$
U(t)=\max _{-L \leq x \leq L}|u(x, t)|
$$

is decreasing in $t$. The solution $u$ represents the entropy in a quasi-static theory of thermoelasticity $[5,6]$, so that Day's results shown that the maximum modulus of the entropy is decreasing in time. In [8] Friedman extended Day's results to a general parabolic equation in n-dimensions of the form

$$
\frac{\partial u}{\partial t}-\sum_{i, j=1}^{n} a_{i, j}(x, t) \frac{\partial^{2} u}{\partial x_{i} \partial x_{j}}+\sum_{i=1}^{n} a_{i}(x, t) \frac{\partial u}{\partial x_{i}}+a u=0
$$

with $a(x, t) \geq 0$ and with the initial and boundary conditions as given in (1.1). Moreover, Friedman proved that there exists $C_{0}>0$ and $\lambda>0$ such that

$$
U(t) \leq C_{0} e^{-\lambda t}, \quad t \geq 0
$$

i.e., $U(t)$ decays to zero exponentially as $t \rightarrow \infty$. Problems similar to the above also arise from the determination of the unknown source parameter [2,9] and other related problems [10].

For physical applications of the problem (1.1), let us consider first the coupled partial differential equations

$$
a \theta_{x x}=b \theta_{t}+\theta_{0} B v_{x x t}, \quad A v_{x x x x}=B \theta_{x x}
$$

which describe the quasi-static flexure of a thermoelastic rod [5]. Here $\theta(x, t)$ is the temperature, $\theta_{0}$ is a uniform reference temperature, $v(x, t)$ is the transverse displacement, $a$ is the conductivity, $b$ is the specific heat at the constant strain, the constant $A$ is the flexure rigidity and the constant $B$ is a measure of the cross-coupling between thermal and mechanical effects. We assume that the ends $x=-L$ and $x=L$ to be maintained at the reference temperture $\theta_{0}$ and to be clamped, that is

$$
\begin{aligned}
\theta(-L, t) & =\theta(L, t)=0, \\
u(-L, t)=u_{x}(-L, t) & =u(L, t)=u_{x}(L, t)=0 .
\end{aligned}
$$

Let

$$
u=\frac{b}{\theta_{0}}\left(\theta-\theta_{0}\right)+B u_{x x}
$$


be the entropy, then after some mathematical manipulations [5] we obtain that $u$ satisfies

$$
a u_{x x}=\left(b+\theta_{0} \frac{B^{2}}{A}\right) u_{t}
$$

with the boundary conditions

$$
\begin{aligned}
u(-L, t) & =-\frac{\theta_{0} B^{2}}{2 b A^{2} L^{2}} \int_{-L}^{L}(L-3 x) u(x, t) d x \\
u(L, t) & =-\frac{\theta_{0} B^{2}}{2 b A^{2} L^{2}} \int_{-L}^{L}(L+3 x) u(x, t) d x
\end{aligned}
$$

and an appropariate initial condition.

For the second example we consider the equations $[1,3,5]$

$$
\begin{aligned}
a \theta_{x x} & =b \theta_{t}+\theta_{0} \alpha(3 \lambda+2 \mu) v_{x t} \\
(\lambda+2 \mu) v_{x x} & =\alpha(3 \lambda+2 \mu) \theta_{x}
\end{aligned}
$$

which describe the behavior of a slab $-L \leq x \leq L$ made of homogeneous and isotropic material. Here $\theta(x, t)$ is the temperature, $v(x, t)$ is the displacement component in the direction of the $x$-axis, $\theta_{0}$ is a uniform reference temperature, $\alpha$ is the coefficient of expansion, and $\lambda, \mu$ are the elastic moduli. The boundary conditions are

$$
\theta(-L, t)=\theta(L, t)=\theta_{0}, \quad v(-L, t)=v(L, t)=0
$$

Let

$$
u=\frac{b}{\theta_{0}}\left(\theta-\theta_{0}\right)+\alpha(3 \lambda+2 \mu) v_{x}
$$

be the entropy, one has [5] that $u$ satisfies

$$
a u_{x x}=b^{*} u_{t}
$$

with the boundary conditions

$$
u(-L, t)=u(L, t)=-\frac{b^{*}-b}{b} \frac{1}{2 L} \int_{-L}^{L} u(x, t) d x
$$

where

$$
b^{*}=b+\theta_{0} \alpha^{2} \frac{(3 \lambda+2 \mu)^{2}}{\lambda+2 \mu} .
$$

For the detail derivations of the above equations we refer to $[1,3,5]$.

The condition (1.2) implies for the first problem that $5 \theta_{0} B^{2}<3 b A^{2}$ and for the second problem that $\frac{b^{*}-b}{b}<1$ or $\theta_{0} \alpha^{2}(3 \lambda-2 \mu)^{2}<(\lambda+2 \mu) b$.

In this article we study finite difference schemes for (1.1). The finite difference procedures proposed below preserve monotonicity, the maximum principle and the exponential decay (if the 
kernel is non-negative) of the solution for equation (1.1); therefore, they are considered as good numerical approximations.

Let $h=\Delta x=\Delta y=1 / N$ for some integer $N>1$, and let $\tau>0$ be a small step-size in time with $t_{n}=n \tau, n=0,1, \cdots$. For a smooth function $v(x, y) \in C^{2}(\bar{\Omega})$ we assume that the following numerical integration formula is valid:

$$
\int_{\Omega} K(x, y, \xi, \eta) v(\xi, \eta) d \xi d \eta=\sum_{m, l=0}^{N} w_{m, l} K\left(x, y, x_{m}, y_{l}\right) v_{m, l}+O\left(h^{2}\right)
$$

where $w_{m, l} \geq 0$ are weights and $v_{m, l}=v\left(x_{m}, y_{l}\right)$ with $x_{m}=m \Delta x, y_{l}=l \Delta y, m, l=0,1, \cdots, N$. For any $0<\rho^{*}<1$, we restrict $h$ to be so small, say for some $h_{0}>0,0<h \leq h_{0}$, that

$$
\sum_{m, l=0}^{N} w_{m, l}\left|K\left(x, y, x_{m}, y_{l}\right)\right| \leq \rho^{*}=\frac{1+\rho}{2}<1, \quad(x, y) \in \Omega .
$$

Here and throughout this paper we assume that $h$ is small enough so that (1.10) is satisfied. In fact (1.10) serves as a discrete version of (1.2). In order to obtain the numerical solution which preserves as many properties of the solution as possible, (1.10) is a necessary and cannot be considered as a constraint on space discretization. For example the weights can be chosen by using trapezoidal rule,

$$
w_{m, l}= \begin{cases}\Delta x \Delta y, & m, l=1,2, \cdots, N-1 \\ \frac{1}{4} \Delta x \Delta y, & m, l \in\{0, N\} \\ \frac{1}{2} \Delta x \Delta y, & \text { otherwise. }\end{cases}
$$

Define the following shorthand notations

$$
\begin{aligned}
\partial_{t} g^{n} & =\frac{g^{n}-g^{n-1}}{\tau}, \\
\Delta^{2} g_{i, j} & =\frac{g_{i+1, j}+g_{i-1, j}+g_{i, j+1}+g_{i, j-1}-4 g_{i, j}}{h^{2}} .
\end{aligned}
$$

We now define our first numerical scheme: Find $\left\{U_{i, j}^{n}\right\}$ such that

$$
\begin{aligned}
& \partial_{t} U_{i, j}^{n}-\Delta^{2} U_{i, j}^{n}=0, \quad i, j=1,2, \cdots, N-1, \quad n \geq 1, \\
& U_{i, j}^{0}=\phi_{i, j}, \quad i, j=0,1,2, \cdots, N \text {, } \\
& U_{i, j}^{n}=K_{i, j}\left(\left\{U_{m, l}^{n}\right\}\right), \quad\{i, j\} \cap\{0, N\} \neq \emptyset, \quad n \geq 1,
\end{aligned}
$$

where

$$
K_{i, j}\left(\left\{U_{m, l}^{n}\right\}\right)=\sum_{m, l=0}^{N} w_{m, l} K\left(x_{i}, y_{j}, x_{m}, y_{l}\right) U_{m, l}^{n}, \quad\{i, j\} \bigcap\{0, N\} \neq \emptyset, \quad n \geq 1
$$

It is clear that (1.12)-(1.13) is a fully-implicit scheme that requires a full-matrix to be solved at each time level due to the boundary integration. For small $h>0$, the matrix will be diagonally-dominant and can be solved by Gaussian-elimination or any standard method. 
Since (1.12)-(1.13) results in an error $O\left(h^{2}+\tau\right)$, we may propose the following numerically economical semi-implicit scheme: Find $\left\{W_{i, j}^{n}\right\}$ such that

$$
\begin{array}{lll}
\partial_{t} W_{i, j}^{n}-\Delta^{2} W_{i, j}^{n}=0, & i, j=1,2, \cdots, N-1, & n \geq 1, \\
W_{i, j}^{0}=\phi_{i, j}, & i, j=0,1,2, \cdots, N, & \\
W_{i, j}^{n}=K_{i, j}\left(\left\{W_{m, l}^{n-1}\right\}\right), & \{i, j\} \cap\{0, N\} \neq \emptyset, \quad n \geq 1 .
\end{array}
$$

Clearly, (1.14) results in an error $O\left(h^{2}+\tau\right)$ and is easy to implement numerically since only a pentdiagonal matrix system needs to be solved at each time level. Therefore, it is a very economical and fast algorithm. In addition, it is also unconditionally stable. Alternative methods, say ADI, may also be used to solve (1.14).

\section{STABILITY, MONONOTONICITY AND EXPONENTIAL DECAY.}

In this section we prove some monotonicity, maximum principle and exponential decay properties for our numerical solutions $U_{i, j}^{n}$ and $W_{i, j}^{n}$. Define

$$
U^{n}=\max _{0 \leq i, j \leq N}\left|U_{i, j}^{n}\right|, \quad W^{n}=0 \leq i, j \leq N \rightarrow \max \left|W_{i, j}^{n}\right|
$$

THEOREM 2.1. Assume that $U_{i, j}^{n}$ is a solution of (1.12)-(1.13) and the initial approximation $U_{i, j}^{0} \not \equiv 0$ for $i, j=1,2, \cdots, N-1$, then the following holds:

$$
0<U^{n}<U^{n-1}, \quad \forall n \geq 1
$$

PROOF. We show that

$$
0<U^{n}<U^{n-1}, \quad \forall n \geq 1
$$

We observe that $U^{n} \geq 0$ for all $n \geq 0$. Consider the first two levels $n=0$ and $n=1$. Assume to the contrary that $U^{0} \leq U^{1}$, then $U^{1}>0$. If $U^{1}=\left|U_{i_{0}, j_{0}}^{1}\right|=U_{i_{0}, j_{0}}^{1}>0$ ( the case $U_{i_{0}, j_{0}}^{1}<0$ can be treated in a similar way) for some $\left(i_{0}, j_{0}\right)$, then it follows from the discrete maximum principle [7] that this maximum is attained at the boundary. Thus $\left(i_{0}, j_{0}\right)$ can be selected to be a boundary point. Then, we see from (1.10), (1.12) and (1.13) that

$$
U^{1}=\left|K_{i_{0}, j_{0}}\left(\left\{U_{m, l}^{1}\right\}\right)\right| \leq \rho^{*} U^{1}
$$

which is impossible unless $U^{1}=0$ since $\rho^{*}<1$. This contradicts $U^{1}>0$. By (1.12), $U^{1}=0$ will lead to $U_{i, j}^{0}=0$ for $i, j=1,2, \cdots, N-1$ which is a contradiction. Thus, $0<U^{1}<U^{0}$.

Now, we consider the levels $n=1$ and $n=2$. By repeating the above argument with $U_{i, j}^{1}$ as the initial data, we can show that $0<U^{2}<U^{1}$. Thus, (2.3) is proved by repeating the above argument for higher levels.

Q.E.D.

THEOREM 2.2. Assume that $W_{i, j}^{n}$ is a solution of (1.14) and the initial approximation $W_{i, j}^{0} \not \equiv 0$ for $i, j=1,2, \cdots, N-1$, then the following holds:

$$
0<W^{n}<W^{n-1}, \quad \forall n \geq 1
$$


PROOF. We show first that $W^{1}>0$. If on the contrary $W^{1}=0$, we will get $W_{i, j}^{0}=0$ for $i, j=1,2, \cdots, N-1$ by (1.14). This contradiction proves $W^{1}>0$. By (1.10), we get

$$
\left|W_{i, j}^{1}\right| \leq \rho^{*} W^{0}<W^{0}, \quad\{i, j\} \bigcap\{0, N\} \neq \emptyset .
$$

According to the discrete maximum priciple [7],

$$
W^{1}=\max _{\{i, j\}} \cap\{0, N\} \neq \emptyset\left|W_{i, j}^{1}\right|<W^{0} .
$$

The remainder of the proof follows from an argument similar to the above and mathematical induction.

REMARK. Theorem 2.1 and Theorem 2.2 imply the unconditionally stability of numerical solutions $U_{i, j}^{n}$ and $W_{i, j}^{n}$, even though $W_{i, j}^{n}$ is the solution of semi-implicit finite difference scheme.

In [8] Friedman proved that $U(t)$ decays exponentially when (1.2) is satisfied. We have proved that both $\left\{U^{n}\right\}$ and $\left\{W^{n}\right\}$ possess the strict monotonicity. In fact numerically there exists $\lambda>0$, as suggested in the examples of section 5, Figure 6 and Figure 7, such that

$$
\log \frac{U^{n+1}}{U^{n}} \sim-\lambda \Delta t \quad \text { as } n \rightarrow \infty
$$

and same is true for $W^{n}$. This motivates the justifications of the exponential decay of $U^{n}$ and $W^{n}$ when the kernel is non-negative.

THEOREM 2.3. Under the assumption that $U_{i, j}^{n}$ is the solution of (1.12)-(1.13) and the kernel $K(x, y, \xi, \eta) \geq 0$, there exists a positive constant $\lambda>0$ such that for $U^{0}=\max _{i, j}\left|U_{i, j}^{0}\right|$,

$$
U^{n} \leq U^{0} e^{-\lambda t_{n}} \text { for all } n \geq 0 \text {. }
$$

PROOF. Let $V(x, y, t)=e^{-\lambda t}\left(2 U^{0}-\frac{e}{4}\left(x^{2}+y^{2}\right)\right)$ where $\epsilon$ and $\lambda$ are two positive constants to be chosen below. It follows easily that there exists $\epsilon_{0}>0$ such that

$$
V(x, y, 0)=2 U^{0}-\frac{\epsilon}{4}\left(x^{2}+y^{2}\right)>U^{0} \text { on } \Omega \text { if } 0<\epsilon \leq \epsilon_{0} .
$$

Also, since $K_{i, j}(\{1\}) \leq \rho^{*}<1$, we find

$$
K_{i, j}\left(\left\{2 U^{0}\right\}\right) \leq \rho^{*} 2 U^{0}<2 U^{0},
$$

and then, there exists a positive constant $\epsilon_{1}>0$ small enough such that for all $0<\epsilon \leq \epsilon_{1}$,

$$
V_{i, j}^{n}>K_{i, j}\left(\left\{V_{m, l}^{n}\right\}\right), \quad\{i, j\} \bigcap\{0, N\} \neq \emptyset, \quad n \geq 1
$$

Thus, we choose $\epsilon=\min \left\{\epsilon_{0}, \epsilon_{1}\right\}$. It follows from a simple calculation that

$$
\partial_{t} V_{i, j}^{n}-\Delta^{2} V_{i, j}^{n}=e^{-\lambda t_{n+1}}\left(\epsilon-\lambda e^{\lambda \xi}\left(2 U^{0}-\frac{\epsilon}{4}\left(x_{i}^{2}+y_{j}^{2}\right)\right), \quad i, j=1,2, \cdots, N-1\right.
$$


where $\xi \in(0, \tau)$. As $\lambda e^{\lambda r} \rightarrow 0$ when $\lambda \rightarrow 0$, we have for some $\lambda_{0}=\lambda_{0}(\epsilon)>0$ ( or $\lambda_{0}=$ $\left.\min \left\{1 / \tau, \epsilon /\left(2 e U^{0}\right)\right\}\right)$ such that for all $0<\lambda \leq \lambda_{0}$,

$$
\partial_{t} V_{i, j}^{n}-\Delta^{2} V_{i, j}^{n}>0, \quad i, j=1,2, \cdots, N-1
$$

Now letting $Z_{i, j}^{n}=V_{i, j}^{n}-U_{i, j}^{n}$ with $\lambda=\lambda_{0}$ and $\epsilon$ chosen above, we see from (1.12) and the analysis above that

$$
\begin{array}{lll}
\partial_{t} Z_{i, j}^{n}-\Delta^{2} Z_{i, j}^{n}>0, & i, j=1,2, \cdots, N-1, & n \geq 1, \\
Z_{i, j}^{0}>0, & j=0,1,2, \cdots, N, & \\
Z_{i, j}^{n}>K_{i, j}\left(\left\{Z_{m, l}^{n}\right\}\right), & \{i, j\} \cap\{0, N\} \neq \emptyset, & n \geq 1 .
\end{array}
$$

We now show that $Z_{i, j}^{n} \geq 0$. Assume that $n_{0}$ is the first level that $Z_{i, j}^{n}$ may take the negative values, then we have $\left(i_{0}, j_{0}\right)$ such that

$$
Z_{i_{0}, j_{0}}^{n_{0}}=\min _{i, j} Z_{i, j}^{n_{0}}<0
$$

It follows from the discrete maximum principle [5] that $\left(i_{0}, j_{0}\right)$ must be the boundary point, otherwise $\partial_{t} Z_{i_{0}, j_{0}}^{n_{0}}-\Delta^{2} Z_{i_{0}, j_{0}}^{n_{0}} \leq 0$ which is not possible. Thus we have from the positivity of kernel $K$ and (2.10) that

$$
-Z_{i_{0}, j_{0}}^{n_{0}}<K_{i, j}\left(\left\{-Z_{m, l}^{n_{0}}\right\}\right) \leq \rho^{*}\left(-Z_{i_{0}, j_{0}}^{n_{0}}\right)
$$

which implies that $Z_{i_{0}, j_{0}}^{n_{0}}=0$, a contradiction. Hence, we have proved that $U_{i, j}^{n} \leq V_{i, j}^{n}$. Using a similar argument by treating $-U_{i, j}^{n}$ it can be shown that $U_{i, j}^{n} \geq-V_{i, j}^{n}$. This completes the proof. Q.E.D.

THEOREM 2.4. Assume that $W_{i, j}^{n}$ is the solution of (1.14), then there exists a positive constant $\lambda>0$ such that $W^{0}=\max _{i, j}\left|W_{i, j}^{0}\right|$,

$$
W^{n} \leq W^{0} e^{-\lambda t_{n}} \quad \text { for all } n \geq 0
$$

PROOF. The proof consists of an argument similar to that given in the proof of Theorem 2.3 , we therefore only give the outline.

Let $V(x, y, t)=e^{-\lambda t}\left(2 W^{0}-\frac{\epsilon}{4}\left(x^{2}+y^{2}\right)\right)$ and as before, let $\epsilon_{0}$ be chosen so small that $V(x, y, 0)>W^{0}$ for $0<\epsilon \leq \epsilon_{0}$. Because the numerical integration uses the data on the previous level for the boundary condition, we need to first select a $\lambda_{0}>0$ such that $0<\lambda \leq \lambda_{0}$,

$$
e^{-\lambda \tau}>\rho^{*}, \quad \text { i.e. } \quad e^{-\lambda \tau} 2 W^{0}>\rho^{*} 2 W^{0} \text {. }
$$

Since $e^{-\lambda r} \rightarrow 1$ when $\lambda \rightarrow 0$, the existence of such a $\lambda_{0}$ is not a problem. With $\epsilon_{0}$ and $\lambda_{0}$ chosen as above, we then select $\epsilon_{1}>0$ so small that for $0<\epsilon \leq \epsilon_{1}$

$$
V_{i, j}^{n}>K_{i, j}\left(\left\{V_{m, l}^{n-1}\right\}\right), \quad\{i, j\} \bigcap\{0, N\} \neq \emptyset, \quad n \geq 1
$$


Using $\epsilon=\min \left\{\epsilon_{0}, \epsilon_{1}\right\}$ and the $\lambda_{0}$ selected above, we select $\lambda_{1}>$ such that for $0<\lambda \leq \lambda_{1}$,

$$
\partial_{\imath} V_{i, j}^{n}-\Delta^{2} V_{i, j}^{n}>0, \quad i, j=1,2, \cdots, N-1
$$

We take $\lambda=\min \left\{\lambda_{0}, \lambda_{1}\right\}$. We omit the reminder of the proof which is the same as that given in the proof of Theorem 2.1 with the $\epsilon$ and $\lambda$ as chosen here.

Q.E.D.

\section{CONVERGENCE AND ERROR ESTIMATES.}

In this section we study the convergence and error estaimes of the numerical procedures proposed in section 1. First, we show the following result.

THEOREM 3.1. Assume that in addition to (1.2), the kernel $K$ in problem (1.1) satisfies $K \in C^{2}(\overline{\partial \Omega \times \Omega})$ and

$$
K(x, y, \xi, \eta) \geq 0, \forall \quad(x, y, \xi, \eta) \in \partial \Omega \times \Omega .
$$

If the solutions $u$ of (1.1) is known apriori to be smooth enough, $u \in C^{4,2}\left(\overline{Q_{T}}\right)$, then there exists a positive constant $C=C\left(\|u\|_{C^{4,2}},\|K\|_{C^{2}}\right)>0$ such that the solution $U_{i, j}^{n}$ of (1.12) satisfies

$$
\max _{i, j, n}\left|U_{i, j}^{n}-u\left(x_{i}, y_{j}, t_{n}\right)\right| \leq C\left(h^{2}+\tau\right)
$$

PROOF. Let $e_{i, j}^{n}=U_{i, j}^{n}-u\left(x_{i}, y_{j}, t_{n}\right)$ for all $i, j, n$, then we see from (1.10) and (1.12) that $e_{i, j}^{n}$ satisfies

$$
\begin{array}{lll}
\partial_{t} e_{i, j}^{n}-\Delta^{2} e_{i, j}^{n}=\tau_{i, j}^{n}, & i, j=1,2, \cdots, N-1, & n \geq 1, \\
e_{i, j}^{0}=0, & i, j=0,1,2, \cdots, N, & \\
e_{i, j}^{n}=K_{i, j}\left(\left\{e_{m, l}^{n}\right\}\right)+\epsilon_{i, j}^{n}, & \{i, j\} \cap\{0, N\} \neq \emptyset, \quad n \geq 1 .
\end{array}
$$

Here $\tau_{i, j}^{n}$ and $\epsilon_{i, j}^{n}$ are the truncation errors induced by the discretization of differential equation and numerical integration respectively. Then there exists $L_{0}>0$ such, that

$$
\max _{i, j, n}\left|\tau_{i, j}^{n}\right| \leq L_{0}\left(h^{2}+\tau\right), \quad \max _{i, j, n}\left|\epsilon_{i, j}^{n}\right| \leq L_{0}\left(h^{2}+\tau\right)
$$

We now define an auxiliary function $\theta(x, y)$ by

$$
\theta(x, y)=\frac{1-x^{2}-y^{2}}{4} L_{0}\left(h^{2}+\tau\right)
$$

then it is easy to verify that

$$
-\Delta^{2} \theta_{i, j}=L_{0}\left(h^{2}+\tau\right) \quad \text { and } \quad 0 \leq \theta_{i, j} \leq \frac{L_{0}}{2}\left(h^{2}+\tau\right) .
$$

Let $Z_{i, j}^{n}=e_{i, j}^{n}-\theta_{i, j}$ for all $i, j, n$, we find from (3.3) and (3.6) that

$$
\begin{array}{lll}
\partial_{t} Z_{i, j}^{n}-\Delta^{2} Z_{i, j}^{n} \leq 0, & i, j=1,2, \cdots, N-1, & n \geq 1, \\
Z_{i, j}^{0}=-\theta_{i, j} \leq 0, & i, j=0,1,2, \cdots, N, & \\
Z_{i, j}^{n}=K_{i, j}\left(\left\{Z_{m, l}^{n}\right\}\right)+K_{i, j}\left(\left\{\theta_{m, l}\right\}\right)-\theta_{i, j}+\epsilon_{i, j}^{n}, & \{i, j\} \cap\{0, N\} \neq \emptyset, & n \geq 1 .
\end{array}
$$


We now show that there exists $C>0$ such that $Z_{i, j}^{n} \leq C\left(h^{2}+\tau\right)$ for all $i, j, n$. If $Z_{i, j}^{n}$ has a positive maximum, then according to the discrete maximum principle then it must be attained at a boundary point. Assume that $M=Z_{i_{0}, j_{0}}^{n_{0}}>0$ with $n_{0} \geq 1$, is the positive maximum. From the boundary condition in (3.7) we see that

$$
\begin{aligned}
M & \leq \rho^{*} M+\left(\rho^{*}+1\right) \max _{i, j}\left|\theta_{i, j}\right|+\max _{i, j, n}\left|\epsilon_{i, j}^{n}\right| \\
& \leq \rho^{*} M+\frac{\rho^{*}+3}{2} L_{0}\left(h^{2}+\tau\right)
\end{aligned}
$$

which implies

$$
M \leq \frac{\rho^{*}+3}{2\left(1-\rho^{*}\right)} L_{0}\left(h^{2}+\tau\right)
$$

Hence, we have proved that

$$
e_{i, j} \leq \theta_{i, j}+\frac{\rho^{*}+3}{2\left(1-\rho^{*}\right)} L_{0}\left(h^{2}+\tau\right) .
$$

If instead $Z_{i, j}^{n}=e_{i, j}^{n}+\theta_{i, j}$, then a similar argument gives

$$
e_{i, j} \geq-\theta_{i, j}+\frac{\rho^{*}+3}{2\left(1-\rho^{*}\right)} L_{0}\left(h^{2}+\tau\right)
$$

Therefore, we find from (3.10)-(3.11) that

$$
\begin{aligned}
\left|e_{i, j}\right| & \leq\left|\theta_{i, j}\right|+\frac{\rho^{*}+3}{2\left(1-\rho^{*}\right)} L_{0}\left(h^{2}+\tau\right) \\
& \leq \frac{3 L_{0}}{1-\rho^{*}}\left(h^{2}+\tau\right),
\end{aligned}
$$

which is (3.1). The proof is complete.

Q.E.D.

THEOREM 3.2. Under the same assumptions of Theorem 3.1, let $W_{i, j}^{n}$ be the solution of (1.14). Then for some positive constant $C>0$, independent of $h$ and $\tau$, we have

$$
\max _{i, j, n}\left|W_{i, j}^{n}-u\left(x_{i}, y_{j}, t_{n}\right)\right| \leq C\left(h^{2}+\tau\right)
$$

PROOF. It follows by a similar argument to that given in the proof of Theorem 3.1. Q.E.D.

REMARK. The error estimates in (3.2) and (3.13) are uniform for all $0 \leq t<\infty$ if $T=\infty$, which is guaranteed by the the condition (1.2).

4. GENERAL SMOOTH KERNEL $K(x, y, \xi, \eta)$.

In this section we consider the effect on the original problem (1.1) when the kernel condition (1.2) is replaced by:

$$
0 \leq K(x, y, \xi, \eta) \leq K_{0}, \quad \forall(x, y, \xi, \eta) \in \partial \Omega \times \Omega
$$


In general if the condition (1.2) is not satisfied, then the numerical procedure of (1.12) or (1.14) may not be stable uniformly for $0<t<\infty$. This will be demonstrated in both theoretically and through numerical examples below. For these kernels, the stability will depend upon $K_{0}$ and $T>0$. Here we consider a class of kernels which satisfy (4.1) but not (1.2).

We first consider the continuous problem. Let $w(x, y)$ be an auxiliary function defined by

$$
1 \leq w(x, y)=1+M\left((x-1 / 2)^{d}+(y-1 / 2)^{d}\right), \quad d>0
$$

where $M$ and $d$ (even) are two positive constants to be chosen. Clearly, we have

$$
\min _{(x, y) \in \partial \Omega} w(x, y)=1+2 M\left(\frac{1}{2}\right)^{d}
$$

Let $u(x, y, t)$ be a solution of (1.1) with $K$ satisfying (4.1), and set Let $v(x, y, t)=\frac{u(x, y, t)}{w(x, y)}$ and find that $v$ satisfies

$$
\begin{aligned}
v_{t} & =\Delta v+2 \frac{\nabla w \cdot \nabla v}{w}+\frac{\Delta w}{w} v, & & \text { in } Q_{T}, \\
v(x, y, 0) & =\frac{\phi(x, y, t)}{w(x, y)}, & & (x, y) \in \Omega \\
v(x, y, t) & =\int_{\Omega} R(x, y, \xi, \eta) v(\xi, \eta) d \xi d \eta, & & (x, t) \in \partial \Omega, \quad t \geq 0,
\end{aligned}
$$

where

$$
R(x, y, \xi, \eta)=K(x, y, \xi, \eta) \frac{w(\xi, \eta)}{w(x, y)}
$$

Thus, we have from (4.1) that

$$
\int_{\Omega}|R(x, y, \xi, \eta)| d \xi d \eta \leq \frac{K_{0}}{1+M(1 / 2)^{d-1}} \int_{\Omega} w(\xi, \eta) d \xi d \eta
$$

A simple calculation shows that if $d$ is an even integer,

$$
\int_{\Omega} w(\xi, \eta) d \xi d \eta=1+M \frac{(1 / 2)^{d-1}}{d+1}
$$

Then it follows that

$$
\int_{\Omega}|R(x, y, \xi, \eta)| d \xi d \eta \leq K_{0} \frac{1+M \frac{(1 / 2)^{d-1}}{d+1}}{1+M(1 / 2)^{d-1}} \rightarrow \frac{K_{0}}{d+1} \quad \text { as } \quad M \rightarrow \infty .
$$

Hence, taking $d=2 K_{0}$ and $M=M\left(K_{0}\right)>0$ large enough, we can achieve

$$
\int_{\Omega}|R(x, y, \xi, \eta)| d \xi d \eta \leq \frac{K_{0}}{d+1} \leq \frac{2 K_{0}}{2 K_{0}+1}<1, \quad \forall \quad(x, y) \in \partial \Omega .
$$

For $w(x, y)$ chosen in this way, we have for some $K_{1}=K_{1}\left(K_{0}\right)>0$ that $|\Delta w / w| \leq K_{1}$.

Now consider the transformation $v(x, y, t)=e^{\lambda t} Y(x, y, t)$ with $\lambda \geq K_{1}$, we find that $Y$ satisfies

$$
\begin{aligned}
Y_{t} & =\Delta Y+2 \frac{\nabla w \cdot \nabla Y}{w}+\left(\frac{\Delta w}{w}-\lambda\right) W & & \text { in } Q_{T}, \\
Y(x, y, 0) & =\frac{\phi(x, y, t)}{w(x, y)}, & & (x, y) \in \Omega \\
Y(x, y, t) & =\int_{\Omega} R(x, y, \xi, \eta) Y(\xi, \eta) d \xi d \eta, & & (x, t) \in \partial \Omega, \quad t \geq 0 .
\end{aligned}
$$


REMARK. We now see from [5] that $Y$ possesses the maximum principle, monotonicity and exponential decay properties, which in turn results in monotonic and stable numerical schemes if it is discretized as (1.12) or (1.14) in an appropriate way.

Turning to numerical approximations for (1.1) with condition (4.1), we let $\tau=T / N_{1}$ where $N_{1}$ is a positive integer. Numerical solutions to the problem, $U_{i, j}^{n}$ or $W_{i, j}^{n}$, are defined as in (1.12) or(1.14). We cannot expect that these two schemes have the monotonic properties as described in Theorem 2.1 and Theorem 2.2 when (1.2) is not satisfied. However, we have the following local stability estimates.

THEOREM 4.1. Assume that $U_{i, j}^{n}$ is defined as in (1.12) or (1.14) for the problem (1.1) with $K \geq 0$ and (4.1) satisfied. If the solution $u$ of (1.1) is known apriori to be smooth enough, $u \in C^{4,2}\left(\overline{Q_{T}}\right)$, then there is some constant $C^{*}=C^{*}\left(\|u\|_{C^{4,2},}\|K\|_{C^{2}}, K_{0}, T\right)>0$ such that

$$
\max _{i, j, n}\left|U_{i, j}^{n}-u\left(x_{i}, y_{j}, t_{n}\right)\right| \leq C^{*}\left(h^{2}+\tau\right)
$$

PROOF. The proof is similar to that given in section 3, so is outlined as below. For (4.11), we let $U_{i, j}^{n}=e^{\lambda t_{n}} w_{i, j} Y_{i, j}^{n}$, where $\lambda$ and $w(x, y)$ are defined as above. Thus, it follows from a simple calculation that $Y_{i, j}^{n}$ satisfies a difference equation which is the discrete version of the equation (4.10). Thus it follows from Theorem 3.1 and Theorem 3.2 (The proof needs only minor modifications from that given in Section 3, we therefore omit.) that there exists a positive constant $C>0$ such that

$$
\max _{i, j, n}\left|Y_{i, j}^{n}-Y\left(x_{i}, y_{j}, t_{n}\right)\right| \leq C\left(h^{2}+\tau\right)
$$

where $C$ is indepedent of $K_{0}$ and $T>0$, and then, we obtain that

$$
\left|U_{i, j}^{n}-u\left(x_{i}, y_{j}, t_{n}\right)\right| \leq e^{\lambda t_{n}} w_{i, j}\left|U_{i, j}^{n}-u\left(x_{i}, y_{j}, t_{n}\right)\right| \leq C^{*}\left(h^{2}+\tau\right)
$$

which completes the proof.

Q.E.D.

REMARK. The constant $C^{*}$ above can be very large if $K_{0}$ and $T>0$ are very large. This can be seen from the choices of $d$ and $K_{1}$ in the above analysis, and also is demonstrated in the examples in section 5 . In another words although $h$ and $\tau$ are small, the error could be very big, even approaching $\infty$ as $n \rightarrow \infty$.

\section{NUMERICAL EXAMPLES.}

We shall report several numerical examples which support our theoretical justifications in the previous sections, i.e., stability, monotonicity and exponential decay as $t \rightarrow \infty$. Both semiimplicit and fully explicit schemes using trapezoidal rule for numerical integration are used in our computations.

EXAMPLE 1. In order to demonstrate the error analysis and stability, we select $\Omega=$ $[0,2 \pi] \times[0,2 \pi], \phi(x, y)=\sin (x) \sin (y)$ and $K(x, y, \xi, \eta)=\frac{k}{4 \pi}$. Thus, for any real constant $k>0$, $u(x, y, t)=\sin (x) \sin (y) e^{-2 t}$ is the solution with $\int_{\Omega}|K(x, y, \xi, \eta)| d \xi \eta=k$. Figure 1 and Figure 2 show by using semi-implicit scheme that the error distributions of $u$ ( the maximum error on each level via the time) with the various parameter $k$ from 0.1 to 4 . Clearly, for $k=0.1,0.3,0.5$ 
and $k=0.8$, even $k=1.0$, the errors are under control as predicted by Theorem 3.1 On the other hand, for $k=1.5,2.5,3$ and $k=4$, it is seen that the errors are under control only for a short period of time, and then divergent to $\infty$ as $n \rightarrow \infty$. This is the exact same result as predicted by Theorem 4.1, i.e., the numerical schemes are stable locally depednent upon $K_{0}>0$ and $T>0$. Figure 3 shows the error distribution of $u$ by using fully implicit scheme. For $0<k<1$ the error distributions of $u$ in this example are almost identical to the case of $k=1$. Also we noticed that the fully implicit scheme is more stable than the semi-implicit scheme.

EXAMPLE 2. We now take a simple model problem with the same spatial domain and kernel as in example $1, \phi(x, y)=\sin (x y)$ and $k=0.8$. Figure 4 and Figure 5 , by using semiimplicit and fully implicit schemes respectively, shows the distribution of $U^{n}$ via the time $t$, which decrease to zero exponentially as $t \rightarrow \infty$. If we assume roughly that for some $\lambda(t), C(t)$ such that

$$
U(t) \sim C(t) e^{\lambda(t) t} \text { as } t \rightarrow \infty
$$

then $\lambda(t)$ can be calculated by the following formula

$$
\lambda^{n} \sim \frac{1}{\Delta t} \log \left(\frac{U^{n+1}}{U^{n}}\right) \text { as } t \rightarrow \infty
$$

Figure 6 and Figure 7, by using semi-implicit and fully implicit schemes respectively, show the distributions of $\lambda(t)$ proposed above, and it is seen that $\lambda^{n}$ approaches to a negative constant as expected. For semi-explicit scheme we find $\lambda^{n} \sim-0.145$, and fully explicit $\lambda^{n} \sim-0.1336$, thus the difference is $1.2 \times 10^{-2}$ which is within the rate of the truncation error of the discretization.

With $\lambda^{n}$ calculated above we then can compute $C(t)$ by

$$
C^{n} \sim U^{n} e^{-\lambda^{n} \Delta t n} \text { as } n \rightarrow \infty
$$

Figure 8 shows the distribution of $C(t)$ computed by semi-implicit scheme according the above assumption. In this example we see that $C(t)$ also approaches to a constant . Figure 9 and Figure 10 are the numerical solutions of $u$ at $t=0.5$ and $t=1.0$ with $h=\pi / 20$ and $\tau=0.01$.

EXAMPLE 3. Taking the same model problem as in example 2 except that the initial data $\phi(x, y)=(\pi-x)(\pi-y)$ and $k=0.4$. Figure 11, Figure 12 and Figure 13 show the distributions of $U(t), \lambda(t)$ and $C(t)$ using the semi-implicit scheme. It is noticed that $U(t)$ goes exponentially to zero very rapidly as $t \rightarrow \infty$ compared to that in example 2, this is due to that $C(t)$ also approaches to zero, not a fixed constant as in example 2.

From these examples We have a rough idea how $U(t)$ will behave as the time advances, i.e., we can at least by using numerical methods, semi-implicit or fully implicit scheme, to estimate the parameter $\lambda$ mentioned in Section 1.

ACKNOWLEDGEMENT: The authors thank Professor S. Riemenschneider for some valuable comments and suggestions which led the improvement of the original manuscript. 


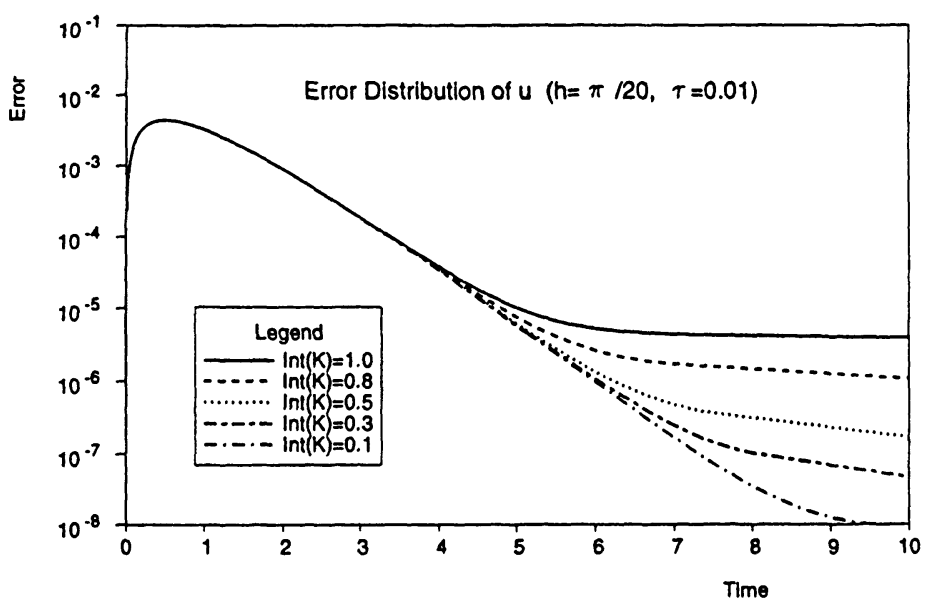

Figure 1: The semi-implicit scheme

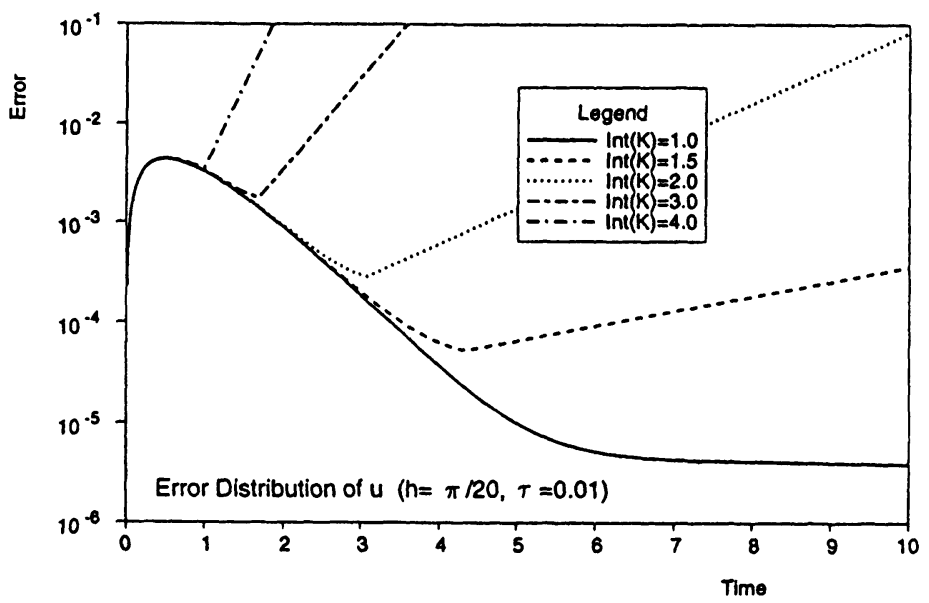

Figure 2: The semi-implicit scheme

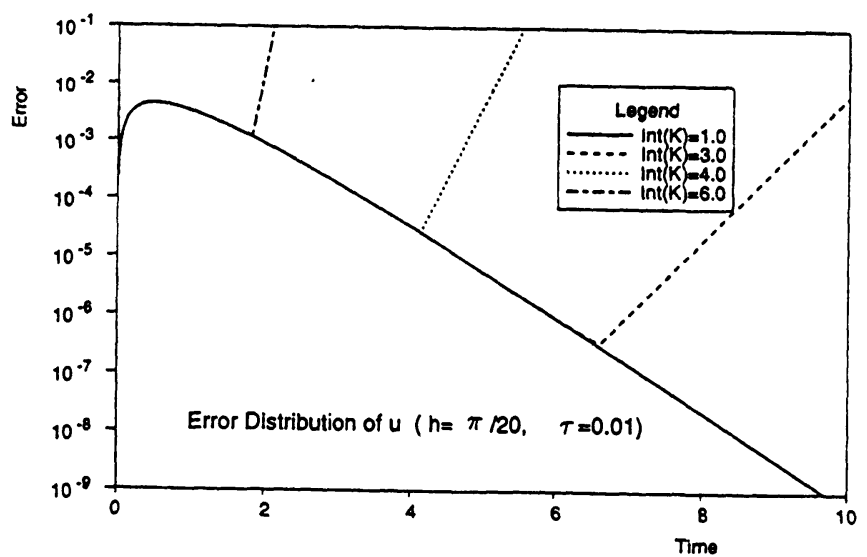

Figure 3: The fully-implicit scheme 


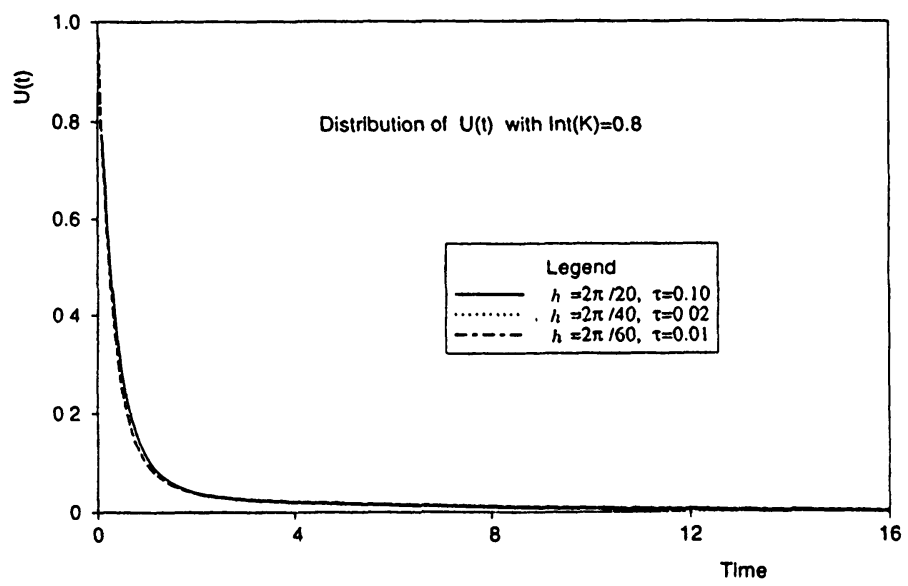

Figure 4: The semi-implicit scheme

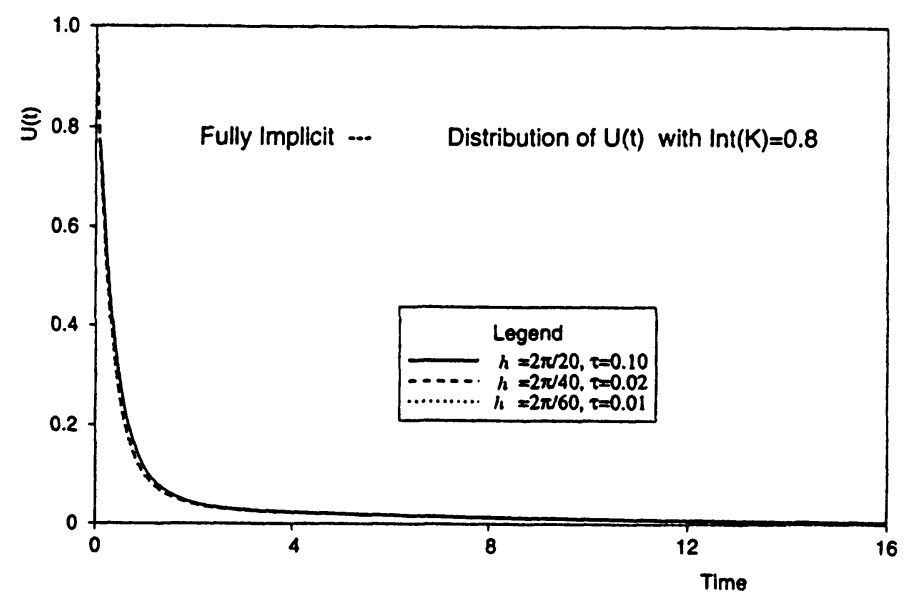

Figure 5: The fully-implicit scheme

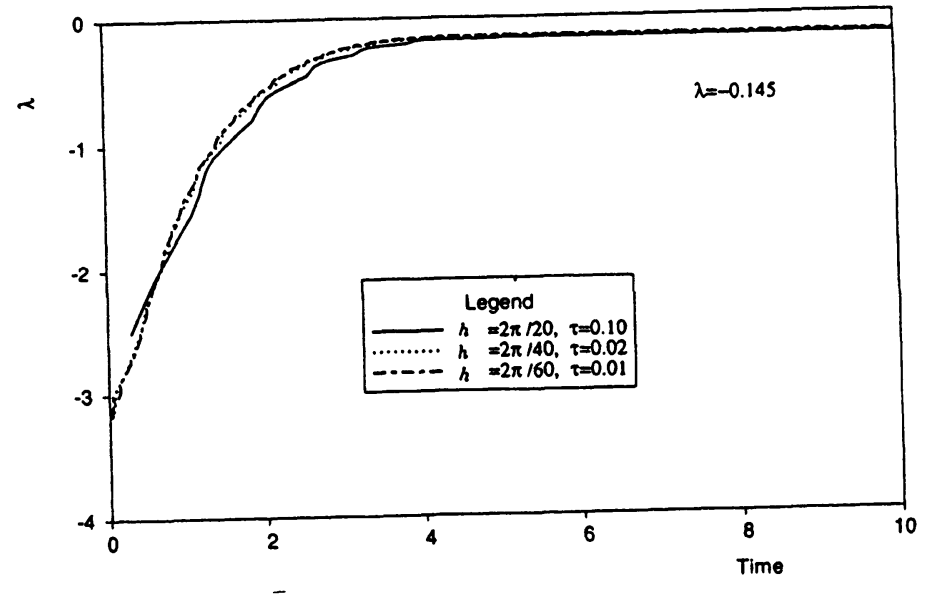

Figure 6: The semi-implicit scheme 


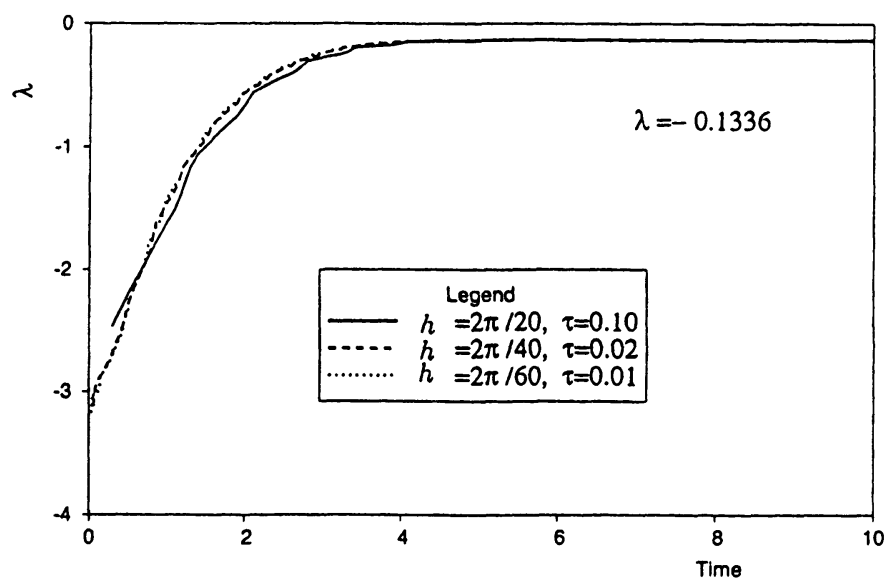

Figure 7: The fully-implicit scheme

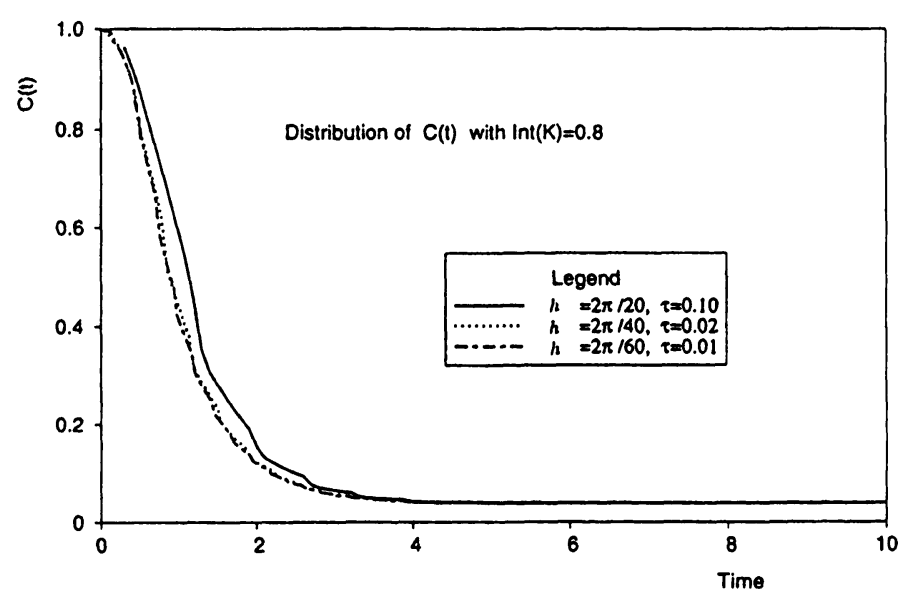

Figure 8: The semi-implicit scheme

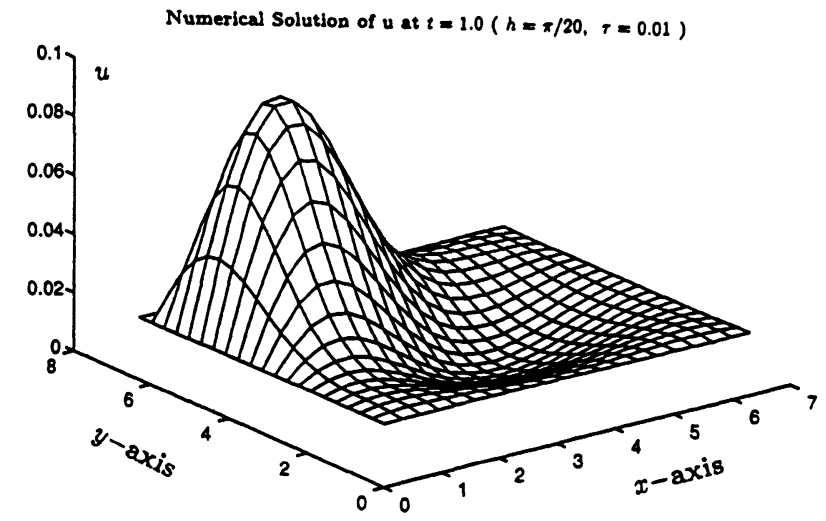

Figure 9: The semi-implicit scheme 


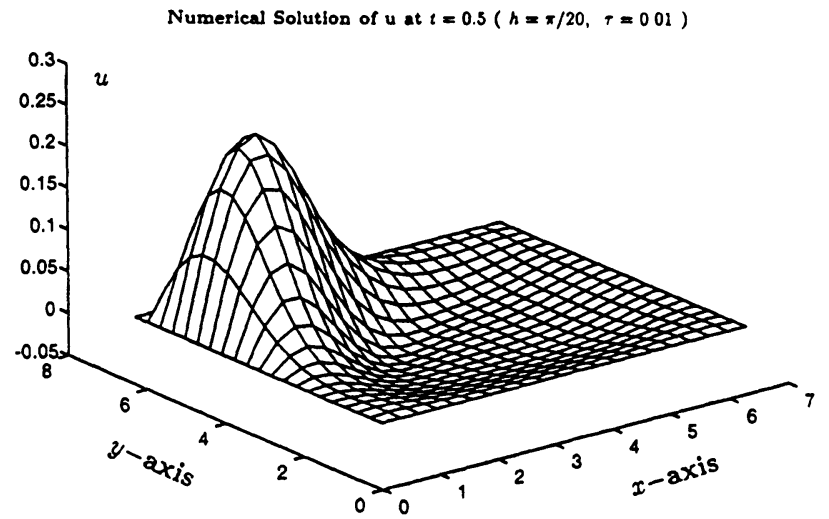

Figure 10: The semi-implicit scheme

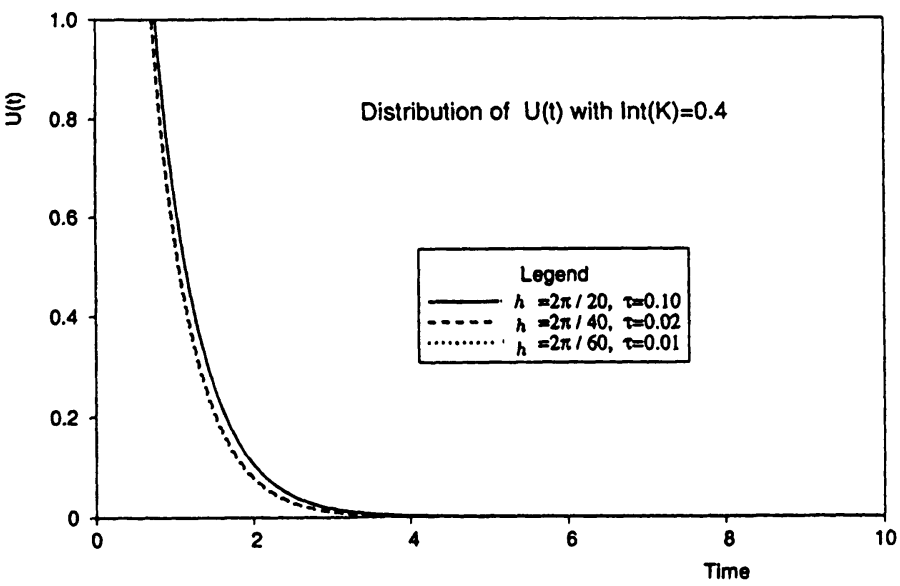

Figure 11: The semi-implicit scheme

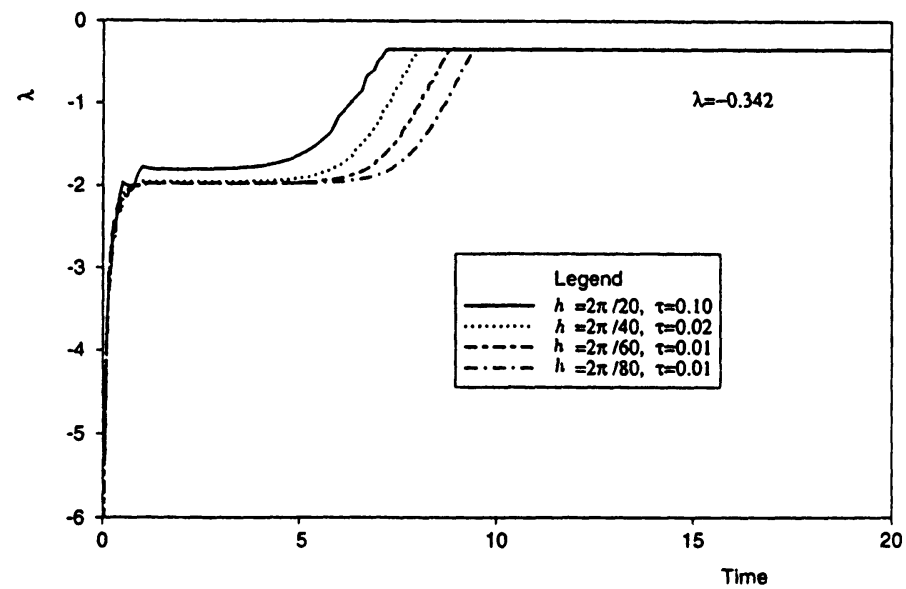

Figure 12: The semi-implicit scheme 


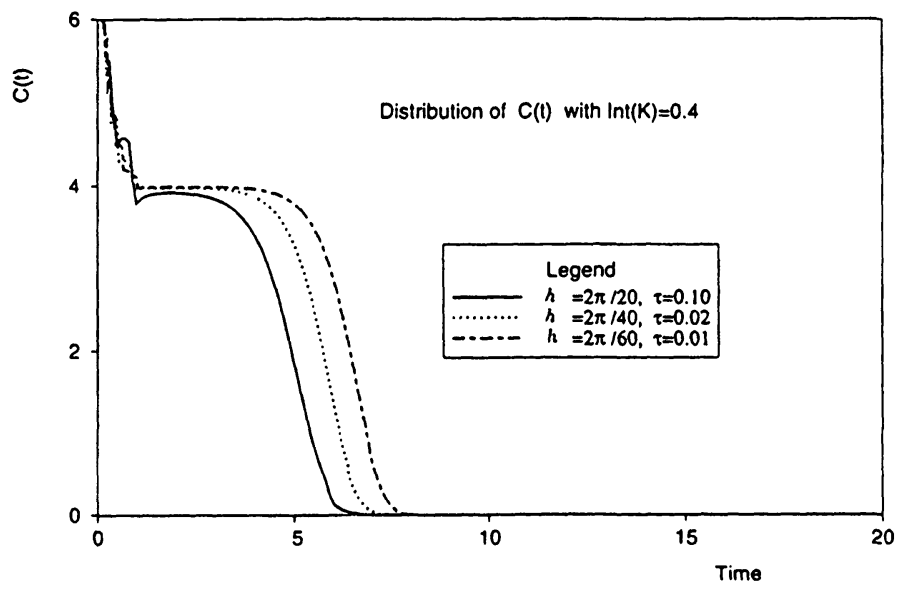

Figure 13: The semi-implicit scheme

\section{REFERENCES}

1. BOLEY, B.A. and WEINER, J.H., Theory of Thermal Stresses, Wiley, New York, 1960.

2. CANNON, J.R., The One-Dimensional Heat Equation, Encyclopedia of Mathematics and Its Applications, 23, Addison-Wesley, Reading, Massachusetts, 1984.

3. CARLSON, D.E., Linear Thermoelasticity, in Encylopedia of Physics, $\underline{2}$, Springer, Berlin, 1972.

4. DAY, W.A., Existence of a property of solutions of the heat equation to linear thermoelasticity and other theories, Q. Appl. Math., $\underline{40}$ (1982) 319-330.

5. DAY, W.A., A decreasing property of solution of a parabolic equation with applications to thermoelasticity, Q. Appl. Math. 41 (1983) 468-475.

6. DAY, W.A., Heat Conduction within Linear Thermoelasticity, Springer-Verlag, New York 1985.

7. J. DOUGLAS Jr., J., On the numerical integration of quasi-linear parabolic equations, Pacific J. Math, $\underline{6}$ (1956) 35-42.

8. FRIEDMAN, A., Monotonic decay of solution of parabolic equations with non-local boundary conditions, Q. Appl. Math., XLIV (1986) 401-407.

9. LIN, Y., An inverse problem for a class of quasilinear parabolic equations, SIAM J. Math. An $\underline{22}$ (1991) 146-156.

10. YURCHUK, N.I., Mixed problem with an integral condition for certain parabolic equations, Diff. Egs., 22 (1986) 2117-2126.

This work is supported in part by NSERC (Canada) and Open Laboratory of CAD/CAM Technology for Advanced Manufacturing, Academic Sinica, No. 9224. 


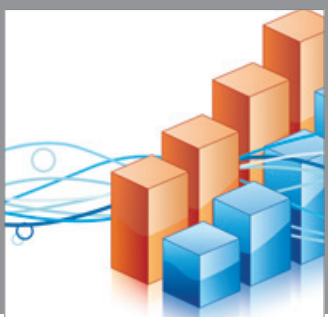

Advances in

Operations Research

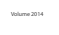

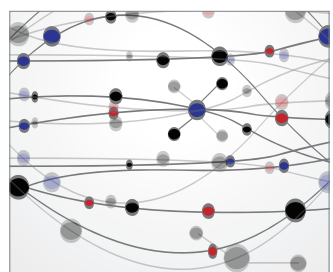

\section{The Scientific} World Journal
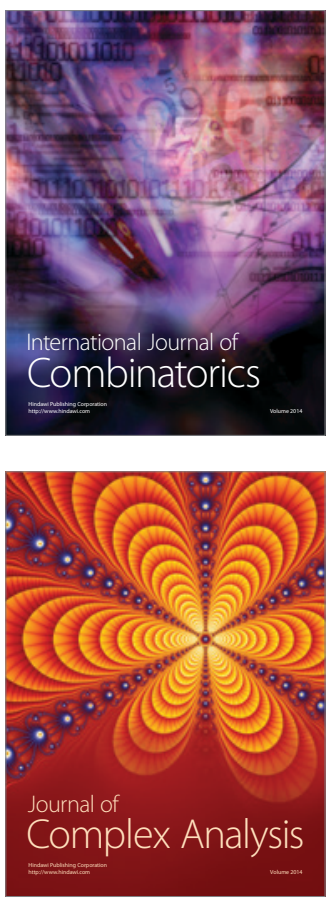

International Journal of

Mathematics and

Mathematical

Sciences
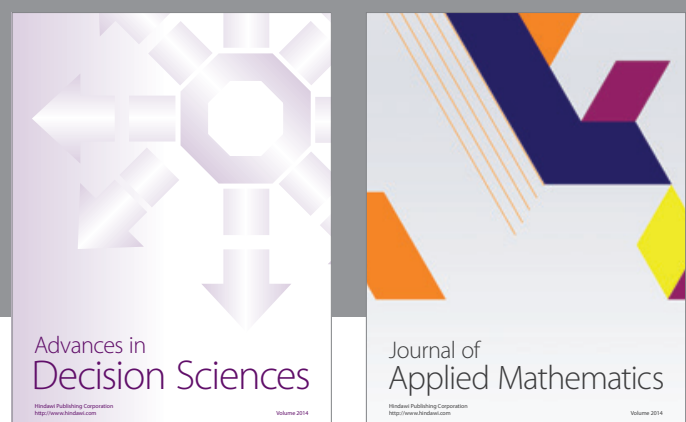

Journal of

Applied Mathematics
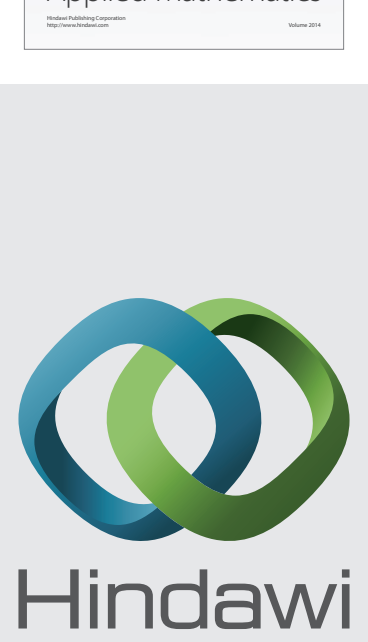

Submit your manuscripts at http://www.hindawi.com
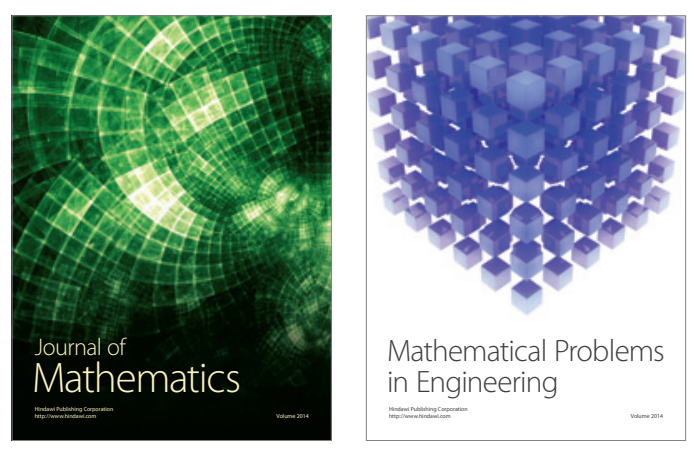

Mathematical Problems in Engineering
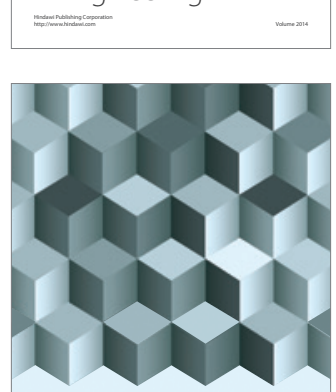

Journal of

Function Spaces
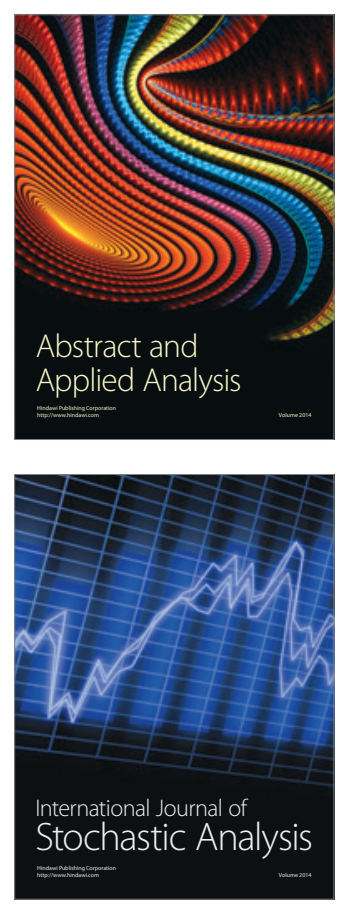

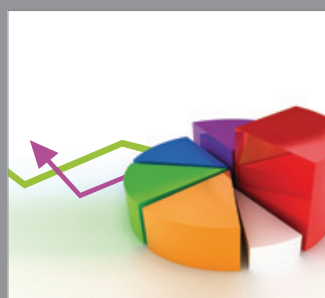

ournal of

Probability and Statistics

Promensencen
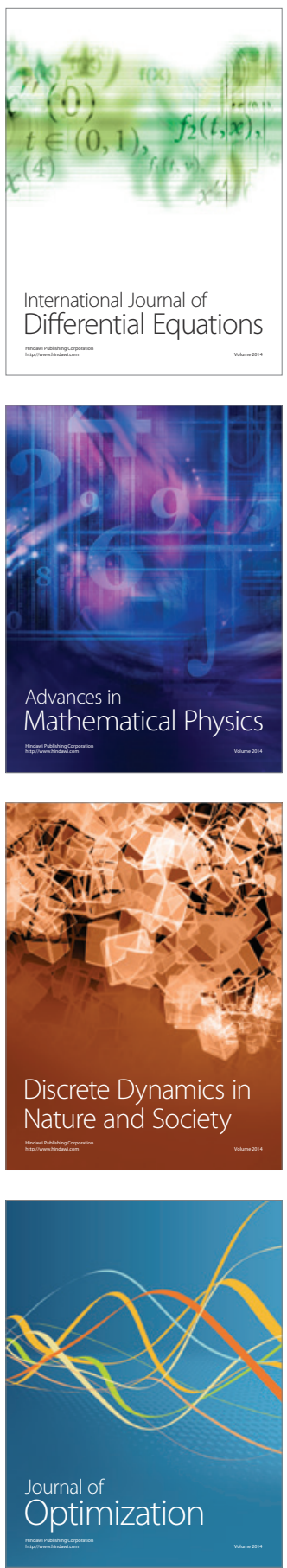